\title{
Sweet plasmonics: Sucrose macrocrystals of metal nanoparticles
}

\author{
Talha Erdem ${ }^{1}$, Zeliha Soran-Erdem ${ }^{1}$, Pedro Ludwig Hernandez-Martinez ${ }^{1,2}$, Vijay Kumar Sharma ${ }^{1}$, Halil Akcali ${ }^{1}$, \\ Ibrahim Akcali ${ }^{1}$, Nikolai Gaponik ${ }^{3}$, Alexander Eychmüller ${ }^{3}$, and Hilmi Volkan Demir ${ }^{1,2}(\varangle)$ \\ ' Departments of Electrical and Electronics Engineering, Physics, UNAM-National Nanotechnology Research Center, and Institute of \\ Materials Science and Nanotechnology, Bilkent University, Ankara 06800, Turkey \\ ${ }^{2}$ School of Electrical and Electronic Engineering and School of Physical and Mathematical Sciences, Nanyang Technological \\ University, Singapore 639798, Singapore \\ ${ }^{3}$ Physical Chemistry, TU Dresden, Bergstrasse 66b, D-01062 Dresden, Germany
}

Received: 6 May 2014

Revised: 18 August 2014

Accepted: 20 August 2014

(C) Tsinghua University Press and Springer-Verlag Berlin Heidelberg 2014

\section{KEYWORDS}

plasmonics, macrocrystals, metal nanoparticles, metal enhanced fluorescence, colloidal quantum dots

\begin{abstract}
The realization of plasmonic structures generally necessitates expensive fabrication techniques, such as electron beam and focused ion beam lithography, allowing for the top-down fabrication of low-dimensional structures. Another approach to make plasmonic structures in a bottom-up fashion is colloidal synthesis, which is convenient for liquid-state applications or very thin solid films where aggregation problems are an important challenge. The architectures prepared using these methods are typically not robust enough for easy handling and convenient integration. Therefore, developing a new plasmonic robust platform having large-scale dimensions without adversely affecting the plasmonic features is in high demand. As a solution, here we present a new plasmonic composite structure consisting of gold nanoparticles (Au NPs) incorporated into sucrose macrocrystals on a large scale, while preserving the plasmonic nature of the Au NPs and providing robustness in handling at the same time. As a proof of concept demonstration, we present the fluorescence enhancement of green CdTe quantum dots (QDs) via plasmonic coupling with these Au NPs in the sucrose crystals. The obtained composite material exhibits centimeter scale dimensions and the resulting quantum efficiency $(\mathrm{QE})$ is enhanced via the interplay between the $\mathrm{Au}$ NPs and CdTe QDs by 58\% (from 24\% to 38\%). Moreover, a shortening in the photoluminescence lifetime from 11.0 to $7.40 \mathrm{~ns}$, which corresponds to a field enhancement factor of 2.4, is observed upon the introduction of Au NPs into the QD incorporated macrocrystals. These results suggest that such "sweet" plasmonic crystals are promising for large-scale robust platforms to embed plasmonic nanoparticles.
\end{abstract}

Address correspondence to volkan@stanfordalumni.org 


\section{Introduction}

Plasmonics attract significant attention in various branches of science and technology including biology [1, 2], physics [3-5], chemistry [6], and photonics [7]. Basically, this field of science deals with the oscillation of free charges on the metallic surfaces. When excited at the correct frequency, these free charges start to oscillate in resonance with the incoming light. In the case of metal nanoparticles (NPs), these oscillating chargescalled localized surface plasmons - can enhance the electromagnetic field locally but strongly, which in turn gives rise to the attractive features of plasmonics such as enhanced absorption, fluorescence, and scattering [3] in addition to improvements of the nonlinear properties [7]. The fluorescence enhancement through localized surface plasmons, about which we present a proof-of-concept study in this report, emanates from the increased radiation probability of near-by emitters as a result of the localized electromagnetic field [8]. Consequently, substantial improvements in the quantum efficiency become possible.

An experimental realization of these improvements associated with plasmonics necessitates metallic structures to be produced with nanometer dimensions, which requires either the use of lithography tools like electron beam and focused ion beam lithography or colloidal synthesis. Among the limitations associated with the lithography approaches, one can count the difficulties and high cost of the fabrication process. In addition, the total size of the samples prepared with these techniques remains generally in the micrometer range, which strongly restricts the use of plasmonic structures in applications requiring large dimensions such as lasers and light-emitting diodes. Furthermore, integration and transfer of these structures to functional devices turn out to be another challenge related to this fabrication method. The colloidal synthesis approach, on the other hand, offers an inexpensive and easy way to obtain metal nanoparticles for plasmonics studies. By carefully selecting the surfactant molecules attached to these nanoparticles, their interaction with other molecules or structures can be easily tailored in liquid environments. Nevertheless, the use of these colloidal nanoparticles in solid state applications is challenging because of the problems in controlling the plasmonic interactions between the metal nanoparticles and other materials. Although the layer-by-layer assembly of nanoparticles enables the preparation of highly controlled films [9], the thickness of the films remains mostly below $100 \mathrm{~nm}$ significantly limiting possible device applications. Methods allowing for preparing films of larger thicknesses like spin coating or drop casting, however, cannot prevent the aggregation of colloidal nanoparticles, which avoids the use of plasmonic interaction in a controlled manner.

As a remedy to these problems, here we present metal nanoparticle embedded sucrose crystals that acquire centimeter scale dimensions and exhibit robust plasmonic character suitable for integration to large scale applications. This material system can be used in various optical systems such as color converting coatings of LED luminaries and color enrichment films of LCD display backlighting units using approaches including powdering and encapsulation in polymers and epoxies that will protect them from external effects like humidity and heat to a certain degree. Furthermore, using pieces of individual macrocrystals, which are selected during or after crystal growth, is another possible simple integration scheme. These sucrose crystals were grown by hybridizing them with gold nanoparticles ( $\mathrm{Au} \mathrm{NPs}$ ) so that the plasmonic nature of the metal nanoparticles is maintained. Furthermore, plasmonic fluorescence enhancement of green CdTe quantum dots (QDs) was demonstrated by hybridizing them with gold nanoparticles within these sucrose crystals (Fig. 1). Different from previous reports of QD embedded macrodimensional salt crystals (i.e., macrocrystals) [10,11], sucrose was deliberately chosen because of the absence of ions in its aqueous solution, since ions in a salt solution strongly trigger aggregation of gold nanoparticles [12]. This causes the plasmonic peak of the absorption spectrum to broaden significantly as soon as $\mathrm{NaCl}$ solution is introduced to the gold nanoparticle dispersion while the color of the mixture turns from pink to gray. As a result, at the end of the crystallization in the presence of salt ions the initial plasmonic peak of gold nanoparticles in the absorption spectrum completely vanishes (see Fig. S1 in the Electronic Supplementary Material (ESM)). Sucrose, 
on the other hand, successfully protected the plasmonic fingerprints of the gold nanoparticles first in the dispersion (Fig. S1 in the ESM) and later in the crystal form (Fig. 2). This hybrid composite material obtained in centimeter scales was shown to increase the quantum efficiency of the green CdTe QDs by $58 \%$ (from $24 \%$ up to $38 \%$ ) via plasmonic coupling as the concentration of $\mathrm{Au}$ nanoparticles increases. In this particular case, the lifetime of the emitter decreased from 11.0 to $7.39 \mathrm{~ns}$ indicating the plasmonic character of the efficiency improvement. Moreover, a further increase of the Au NP content, while keeping the QD content the same, caused strong quenching of quantum efficiency down to $4.6 \%$ together with an increase in the lifetime up to $12.6 \mathrm{~ns}$. By using the information about quantum efficiency and photoluminescence lifetimes, we carried out a detailed theoretical analysis to identify the origin of the changes in the photophysical features of the macrocrystals. We found out that the increase of the field enhancement factor plays a crucial role in the fluorescence enhancement. On the other hand, the fluorescence quenching was attributed to the decrease of the field enhancement factor below unity, as also reported by Teng et al. [13] while the effect of nonradiative energy transfer from CdTe QDs to Au NPs remained weaker. Bearing in mind its simplicity, inexpensiveness, and robustness, we believe that this new composite material may find applications in various fields of

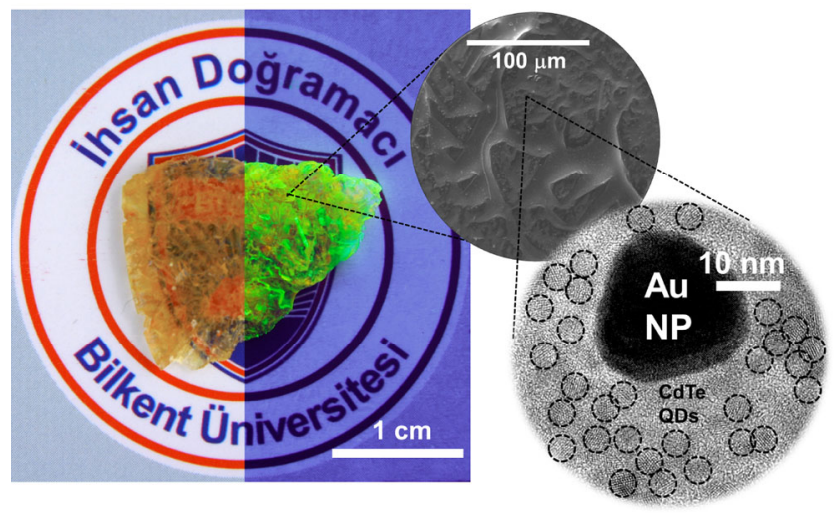

Figure 1 True color images of CdTe QDs and Au NPs embedded in macrodimensional sucrose crystals, which show an efficiency enhancement of $58 \%$ through plasmonic interaction, under ambient lighting and ultraviolet illumination along with scanning electron microscopy (middle) and transmission electron microscopy (right) images.
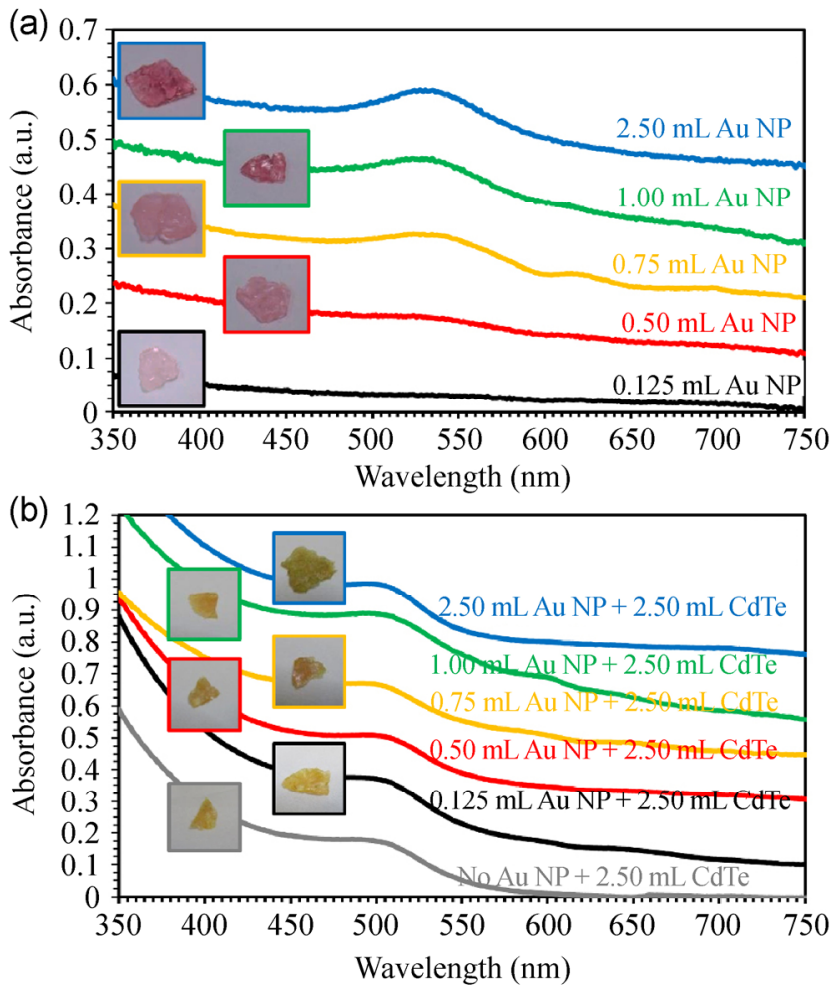

Figure 2 Absorption spectra and true color photographs of the macrocrystals incorporating Au NPs (a) alone and (b) co-immobilized together with CdTe QDs.

science, especially in optics and photonics, requiring solid materials with large dimensions that employ plasmonic interaction.

\section{Results and discussion}

The as-synthesized CdTe QDs exhibited a peak emission wavelength of $523 \mathrm{~nm}$, while their first exciton band in the absorption spectrum was located at $476 \mathrm{~nm}$ (Fig. S2 in the ESM). In the absence of the metal NPs, the emission peak of CdTe QDs in the sucrose macrocrystals shifted to $\sim 534 \mathrm{~nm}$. Furthermore, a clear plasmon peak was monitored at $522 \mathrm{~nm}$ in the absorption spectrum of the Au NPs in water (Fig. S2 in the ESM). After mixing the Au NP dispersion with sucrose solution, the plasmonic features of the nanoparticles were retained except for a slight red-shift and a slightly enhanced absorption (Fig. S1 in the ESM). When Au NPs were embedded into the sucrose crystals, the plasmonic absorption peak was observed to shift further to $532 \mathrm{~nm}$ (Fig. 2).

This red-shift and absorption enhancement in the 
absorption peak of the Au NP is attributed to the difference of the refractive indices of water $\left(n_{\text {water }}=\right.$ $1.33)$ and sucrose crystal $\left(n_{\text {sucrose }}=1.56\right)$ [14], and was confirmed using the simulation tool of extinction, scattering and absorption efficiencies of single and multilayer nanoparticles at http://nanohub.org. The absorption spectra of the sucrose crystals with and without CdTe QDs and with different amounts of $\mathrm{Au}$ NPs are presented in Fig. 2.

In this proof-of-concept demonstration, these $\mathrm{Au}$ NPs and CdTe QDs were intentionally chosen so that the plasmonic peak of the Au NP absorption and photoluminescence of QDs overlap to reveal a strong plasmonic interaction in this composite material system. Nonetheless, there is no physical or chemical restriction on applying this system to other organic and inorganic emitters and metal NPs of other sizes, shapes, and materials, which further widens its applicability.

We observe that the plasmonic absorption peaks for low concentrations of Au NPs are barely recognizable; however, they appear very strongly when the Au NP content is further increased. This observation shows that $\mathrm{Au}$ NPs retain their plasmonic features within the sucrose crystals as opposed to the case of embedding them in salt macrocrystals, where we observed a distinct color change of the mixture from pink to gray immediately after the preparation of the mixture due to the aggregation of $\mathrm{Au} \mathrm{NPs}$ in the presence of positive ions (Fig. S1 in the ESM). On the other hand, when the Au NPs and CdTe QDs are crystallized together with sucrose, the absorption spectra do not reveal any qualitative differences compared to the crystals without Au NPs. However, the bumps at longer wavelengths that occur due to the interaction of Au NPs with each other can still be observed. The true-color photographs of Au NP embedded crystals in this figure clearly show that the pink color of Au NPs associated with the plasmonic effect is maintained in sucrose crystals. To further confirm that the metal nanoparticles in sucrose crystals preserve their plasmonic character without severe aggregation, $\sim 15 \mathrm{mg}$ of $2.50 \mathrm{~mL} \mathrm{Au} \mathrm{NP}$ incorporated sucrose crystal was re-dissolved in $5 \mathrm{~mL}$ of water. The absorption spectrum of this dissolved crystal (Fig. S3 in the ESM) indicates that the plasmonic peak retains its position in spite of a slight broadening, which suggests that the permanent aggregation of $\mathrm{Au}$ NPs in sucrose remains limited.

Subsequent to the absorption of the macrocrystals, their fluorescence quantum efficiency was measured to be $24 \%$ for the sample with no Au NPs and $2.50 \mathrm{~mL}$ (352.5 nmol) CdTe QDs while the quantum efficiency of the CdTe QDs is $20 \%$ in water (for the sake of simplicity in reading, volumes used in the crystallization are preferred to particle amounts throughout the text). This increase in the quantum efficiency was also observed in the work of Rogach et al., and possibly stems from the better surface passivation of QDs within the crystals [11]. For the cases of $0.125(0.39 \mathrm{pmol})$ and $0.50 \mathrm{~mL}(1.56 \mathrm{pmol})$ of $\mathrm{Au} \mathrm{NP}$ incorporation together with $2.50 \mathrm{~mL}$ (352.5 nmol) CdTe QDs, we observed enhanced quantum efficiencies of $29 \%$ and $38 \%$, respectively. In other words, the respective quantum efficiency enhancement of the QDs was $21 \%$ and $58 \%$ for these two cases. Further increase of the Au NP amount caused a decrease in the quantum efficiency of the macrocrystals, as also suggested on the basis of theoretical studies by Govorov et al. [15], to $22 \%$, $4.6 \%$, and $6.9 \%$, respectively, when 0.75 (2.34 pmol), $1.00(3.13 \mathrm{pmol})$, and $2.50 \mathrm{~mL}(15.6 \mathrm{pmol})$ of $\mathrm{Au} \mathrm{NP}$ were employed together with $2.50 \mathrm{~mL}(352.5 \mathrm{nmol})$ CdTe QDs. The quantum efficiency corrected photoluminescence spectra belonging to these macrocrystals are presented in Fig. 3 along with the measured quantum efficiencies. Analysis of Fig. 3 reveals that the emission of the macrocrystals around the plasmonic peak of the Au NP was increased compared to the other parts of the emission spectrum for the crystals having fluorescence enhancement. This observation indicates that the enhancement in emission is very likely to stem from the localized surface plasmons of $\mathrm{Au}$ NPs. For crystals experiencing quenching, the emission spectrum should exhibit a decrease in the emission intensity around the same wavelength range $(\sim 532 \mathrm{~nm})$. However, such an observation is not apparent in the spectra belonging to the corresponding crystals.

At this point, a lifetime analysis is crucial to clearly 
(a)

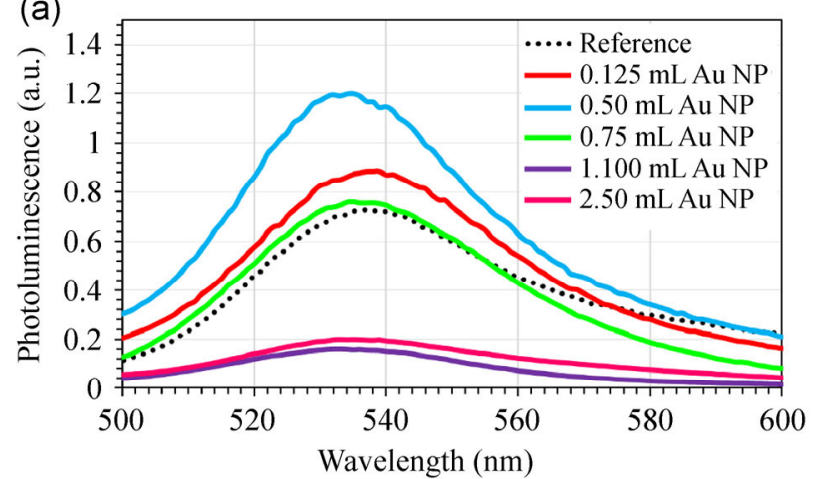

(b)

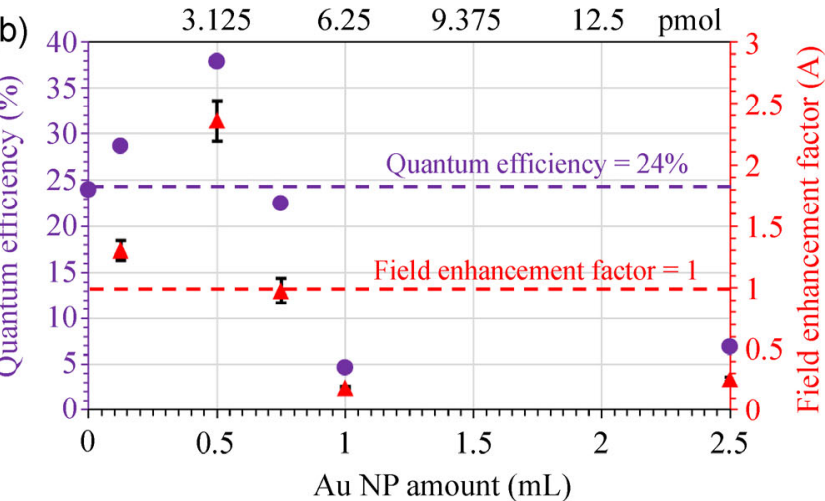

Figure 3 (a) Quantum efficiency corrected photoluminescence spectra of the macrocrystals containing various amounts of Au NPs and $2.5 \mathrm{~mL}$ (352.5 nmol) of CdTe QDs, and (b) quantum efficiencies (purple circles) and the field enhancement factors (red triangles) of the corresponding macrocrystals. The purple dashed line indicates the quantum efficiency level when no Au NPs are present in the macrocrystal while the red dashed line stands for a field enhancement factor of unity.

identify changes in the quantum efficiency when $\mathrm{Au}$ NPs are introduced into the macrocrystals. For this purpose, we measured the time resolved photoluminescence decays of the composite macrocrystals and revealed the lifetime kinetics (Fig. 4 and Table S1 in the ESM). The results show that the lifetimes are shortened compared to the case of no metal NPs present when there is fluorescence enhancement, while the opposite is the case when there is fluorescence quenching. The reference sample containing no $\mathrm{Au}$ NPs but prepared using $2.50 \mathrm{~mL}$ of CdTe QDs exhibited a photoluminescence lifetime of $11.0 \mathrm{ns,}$ while the lifetimes of 0.125 and $0.50 \mathrm{~mL} \mathrm{Au} \mathrm{NP}$ and the same amount of QD including macrocrystals (those experiencing fluorescence enhancement) became 10.1 and $7.39 \mathrm{~ns}$, respectively. $0.75,1.00$, and $2.50 \mathrm{~mL}$ $\mathrm{Au}$ NP incorporated macrocrystals, which experienced fluorescence quenching, possessed lifetimes of 10.6, 11.6, and $12.6 \mathrm{~ns}$ respectively. These observations together with the increase in quantum efficiency can be explained in terms of plasmonic interactions between the QDs and the Au NPs, and the results are strongly parallel to the study of Teng et al. [13], where lifetime shortening (lengthening) was observed in the case of plasmonic fluorescence enhancement (quenching) of Eosin $\mathrm{Y}$ on gold nanostructures fabricated employing electron beam lithography. In our experiment, only the sample including $0.75 \mathrm{~mL}$ of $\mathrm{Au}$ NP does not obey the trend indicated above. However, the quantum efficiency and lifetime of this sample turned out to be very close to the sample containing no metal nanoparticles, therefore, also the $0.75 \mathrm{~mL}$ metal nanoparticle containing macrocrystals can be regarded as obeying the same trend.

For a deeper understanding of the system, we can start the analyses of the results with the definition of quantum efficiency that is the ratio of the radiative recombination rate $\left(\gamma_{\mathrm{r} 0}\right)$ to the sum of the radiative and nonradiative recombination $\left(\gamma_{\mathrm{nr} 0}\right)$ rates in the absence of the metal nanoparticles (Eq. (1))

$$
\eta_{0}=\frac{\gamma_{\mathrm{r} 0}}{\gamma_{\mathrm{r} 0}+\gamma_{\mathrm{nr} 0}}
$$

When there is a metal nanoparticle in the proximity of the emitter, interactions start to occur between the emitter and metal nanoparticle. The electric field $(E)$ around the metal nanoparticle is enhanced upon the generation of localized surface plasmons, and this strengthened field is felt by the nanoparticle whose radiative recombination rate $\left(\gamma_{\mathrm{r}}\right)$ is modified as $\gamma_{\mathrm{r}}=A \gamma_{\mathrm{r} 0}$ where $A$ is the field enhancement factor defined by the ratio of the total electric field intensity of the emitter in the presence ( $\left.E_{\mathrm{MNP} \text { present }}\right)$ and absence ( $\left.E_{\mathrm{MNP} \text { absent }}\right)$ of metal nanoparticles (Eq. (2)).

$$
A=\frac{\int\left|E_{\text {MNP present }}\right|^{2} \mathrm{~d} V}{\int\left|E_{\text {MNP absent }}\right|^{2} \mathrm{~d} V}
$$

Furthermore, the interaction of the emitter and metal nanoparticle opens another path for the excitons generated in the QD from the QDs to the 

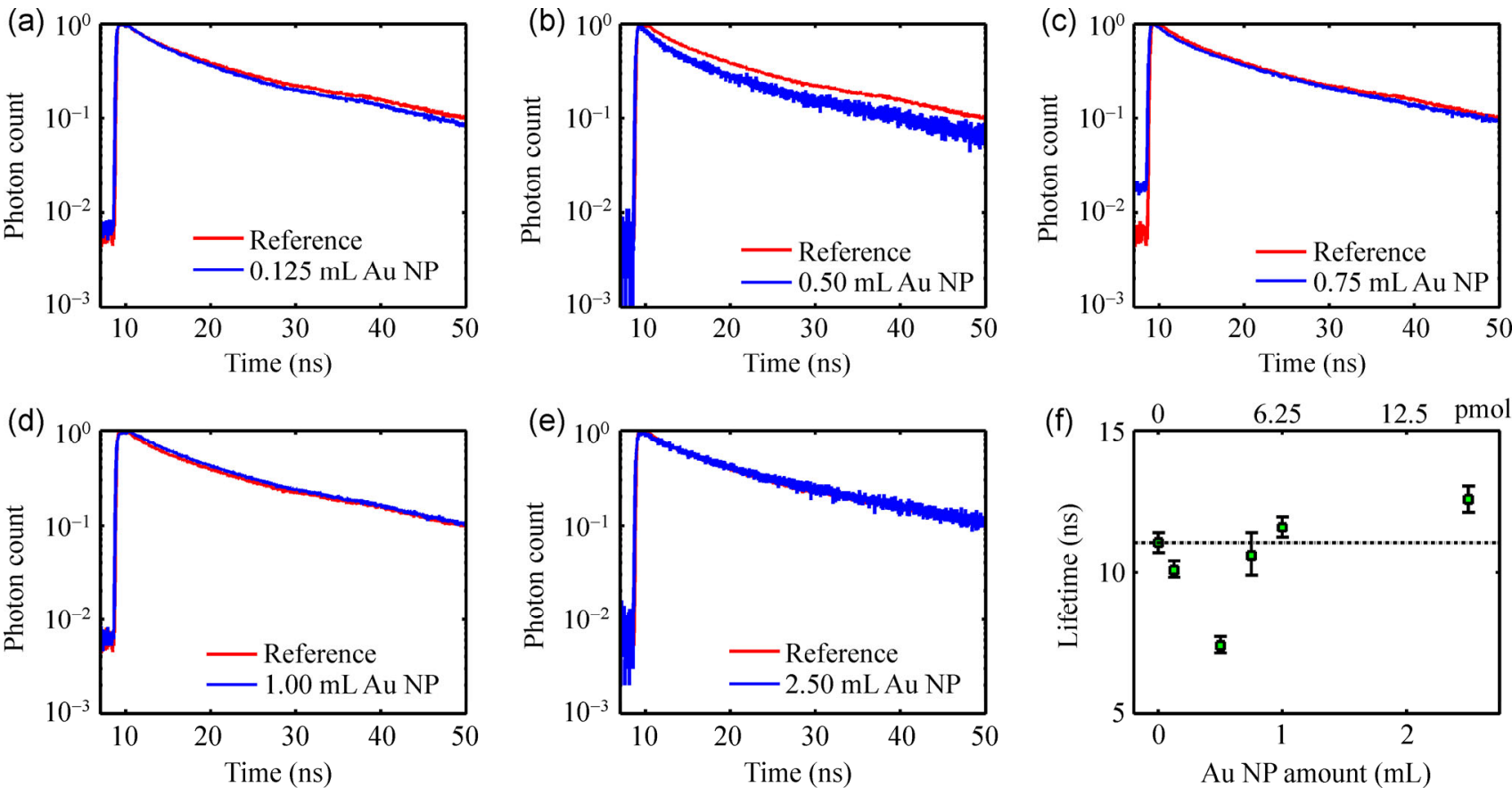

Figure 4 (a)-(e) Time-resolved fluorescence decays of CdTe QDs embedded in plasmonic macrocrystals. All of the crystals were grown using $2.50 \mathrm{~mL}$ of CdTe QDs, and the Au NP amounts were increased from 0.125 to $2.50 \mathrm{~mL}$. The reference sample does not include any Au NPs. (f) The lifetimes of the macrocrystals as a function of the Au NP amount used in the crystallization. Black dashed line indicates the lifetime when no Au NPs are present in the macrocrystal.

metal nanoparticles as a nonradiative energy transfer $\left(\gamma_{\text {nr,metal }}\right)$. Assuming that the intrinsic nonradiative channels are not affected as suggested by Govorov et al. [15], the total recombination rate and fluorescence quantum efficiency are modified in the presence of metal nanoparticles as in Eqs. (3) and (4), respectively

$$
\begin{gathered}
\gamma_{\text {total }}=A \gamma_{\mathrm{r} 0}+\gamma_{\mathrm{nr} 0}+\gamma_{\mathrm{nr}, \text { metal }} \\
\eta=\frac{A \gamma_{\mathrm{r} 0}}{A \gamma_{\mathrm{r} 0}+\gamma_{\mathrm{nr} 0}+\gamma_{\mathrm{nr}, \text { metal }}}
\end{gathered}
$$

In our experiments, we observed that in the case of fluorescence enhancement $\left(\eta>\eta_{0}\right), \gamma_{\text {total }}>\gamma_{\text {total, } 0}$ where $\gamma_{\text {total }, 0}=\gamma_{\mathrm{r} 0}+\gamma_{\mathrm{n} r 0}$; and $\gamma_{\text {total }}<\gamma_{\text {total, } 0}$ in the case of fluorescence quenching $\left(\eta<\eta_{0}\right)$. When the fluorescence enhancement is observed, the field enhancement factor $A$ should be larger than unity so that the ratio of $A \gamma_{\mathrm{r} 0}$ to the total decay rate $\gamma_{\text {total }}$ increases. As a result, an increase in the total recombination rate should be observed which confirms our experimental findings stated above. On the other hand, the fluorescence quenching can be realized with an increase in the total recombination rate if this increase is larger than the increase of the radiative recombination rate. Nevertheless, our experimental results indicate a different behavior; in our case, the total recombination rate decreases. This can only happen if the field enhancement factor decreases below unity which is the case in our study (Fig. 3(b)) and was explained theoretically in terms of the dynamic screening effect in Ref. [15]. An investigation of $\gamma_{\text {nr,metal }}$ together with $A$ revealed that the decrease of the total decay rate in the case of fluorescence quenching was mainly driven by the decrease of $A$ below unity. Our calculations showed that the decrease in the radiative emission rate cannot be compensated by the increase of nonradiative energy transfer rate; as a result, we observe a strong lifetime lengthening when the quantum efficiency decreases strongly.

Since plasmonic coupling is strictly distance dependent [15], the plasmonic interaction between the metal NPs and QDs in the dispersion is expected to be much weaker due to the increased interparticle distance in dispersion compared to the case of solid crystals. To reveal this effect, we investigated 
the emission dynamics of CdTe QDs in the dispersion mixture using which the crystallization was carried out by employing time-resolved fluorescence. As expected, the fluorescence decay curves presented in Fig. S3 in the ESM indicated no significant change in the QD emission dynamics upon the inclusion of the metal nanoparticles in the dispersion. This confirms that the interaction between metal nanoparticles and quantum dots is strongly distance dependent and strengthens in solid crystals as a result of decreased interparticle distance.

The structural characterizations of the composite macrocrystals were carried out using scanning electron microscopy (SEM), transmission electron microscopy (TEM), and X-Ray diffraction (XRD). The SEM image presented in Fig. 5(a) shows that the composite structure has clear crystal facets with varying sizes. On the other hand, the TEM image shown in Fig. 5(b) indicates that the CdTe QDs and the Au NPs are in close proximity which is necessary for the plasmonic interaction. In Fig. 5(b), some of the QDs are marked with black circular indicators for visual assistance. To reveal the general distribution of the QDs in the matrix, the same figure is presented without black circles in Fig. S4 (in the ESM). Figure 5(b) shows that the QDs may form small aggregates in some regions. However, the optical characterizations reveal that this aggregation does not cause a net decrease in the quantum efficiency of the QDs mainly because of a better surface passivation of the QDs [11] and the plasmonic interaction.

The crystalline structure of the sucrose macrocrystals with embedded CdTe QDs and Au NPs was investigated using XRD (Fig. 6). The crystal structure

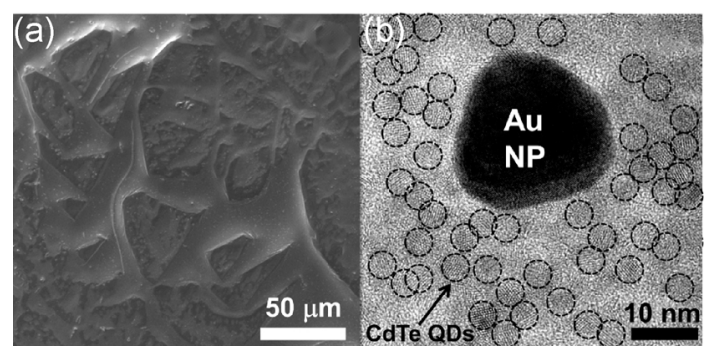

Figure 5 (a) SEM and (b) TEM images of the Au NP and CdTe QD incorporated macrocrystals belonging to the macrocrystal prepared using $0.50 \mathrm{~mL} \mathrm{Au} \mathrm{NPs} \mathrm{and} \mathrm{2.50} \mathrm{mL} \mathrm{CdTe} \mathrm{QDs.} \mathrm{Some}$ of the QDs in the TEM image are marked with dashed black circles for visual assistance.
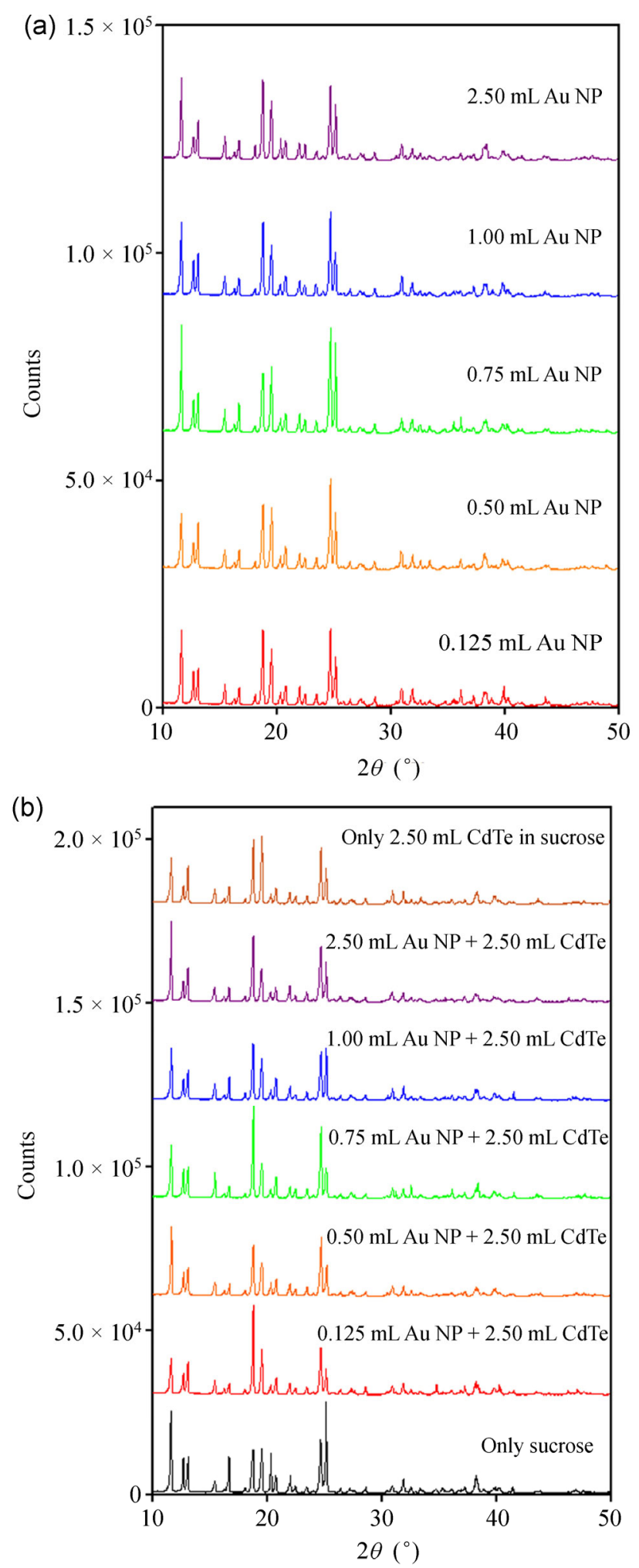

Figure 6 XRD spectra of the composite crystals: (a) XRD spectra of the sucrose macrocrystals having only Au NPs and (b) the spectra of the sucrose macrocrystals with only CdTe incorporated, and the ones grown using mixtures of Au NPs and CdTe QDs. 
of sucrose was identified as monoclinic (JCPDS\#241977) and polycrystalline. We observe that the crystallinity of sucrose decreases with the addition of $\mathrm{Au}$ NPs and/or CdTe QDs. The prominent peaks of sucrose at $11.6^{\circ}, 16.7^{\circ}, 20.4^{\circ}$, and $25.2^{\circ}$ decrease with the addition of Au NPs and/or CdTe QDs. It was also observed that some orientations (resulting in increased peak intensities at $d=4.53$ and $5.70 \AA$ ) of sucrose are preferred over others when Au NPs and/or CdTe QDs were added. We do not observe any shift in the sucrose crystal peaks after addition of Au NPs and/or CdTe QDs, which make us think that the macrocrystals are simply a mixture of sucrose, Au NPs, and CdTe QDs. Furthermore, we did not observe any peaks associated with Au NPs and/or CdTe QDs in the XRD spectra. This may be due to the low concentration of $\mathrm{Au}$ NPs and/or CdTe QDs in the sucrose macrocrystals, since XRD is not sensitive enough to detect material concentrations of less than $\sim 2$ at. $\%$.

\section{Conclusion}

We have demonstrated a robust and large scale plasmonic composite material system suitable for the integration into various optoelectronic devices. By incorporating gold nanoparticles into sucrose macrocrystals, we showed that the final structure still exhibits the plasmonic features of the metal nanoparticles as opposed to embedding in salts. As a proof-of-concept demonstration of plasmonic fluorescence enhancement in macrocrystals, we also hybridized gold nanoparticles with CdTe quantum dots and co-immobilized them within sucrose crystals. As a result of the plasmonic interplay between the metal nanoparticles and the QDs, the quantum efficiency of the emitter particles was improved by $58 \%$ at maximum. The plasmonic character of this enhancement was further verified by the time-resolved fluorescence analyses indicating a lifetime shortening from 11.0 to $7.39 \mathrm{~ns}$. Further increase of the $\mathrm{Au} \mathrm{NP}$ content in the macrocrystal caused quenching in the quantum efficiency, which through theoretical analyses was attributed to the decrease of the field enhancement factor below unity due to screening effect rather than the nonradiative energy transfer from the QDs to metal nanoparticles. We believe that the results presented in this work can open up the way to novel robust device architectures having large dimensions that employ plasmonic interactions as a remedy to the dimension restrictions of conventional fabrication and/or assembly methods.

\section{Materials and methods}

\subsection{Synthesis of CdTe QDs and Au NPs}

The synthesis of CdTe QDs was carried out according to the well-established synthesis procedure of Gaponik et al. [16]. Details of a typical synthesis are presented in the ESM including the absorption and photoluminescence spectra of the synthesized QDs (Fig. S2). The size and concentration of the QDs were calculated according to Ref. [17] as $\sim 1.8 \mathrm{~nm}$ and $141 \mu \mathrm{M}$, respectively.

The gold nanoparticles used in this study were synthesized using the citrate reduction approach [18]. Details of the synthesis and the absorption spectrum of Au NPs are presented in Fig. S2 in the ESM. The size and concentration of the as-synthesized Au NPs were calculated according to Ref. [19] and found to be $\sim 15 \mathrm{~nm}$ and $2.5 \mathrm{nM}$, respectively.

\subsection{Growth of sucrose crystals}

Sucrose stock solution for the crystallization studies was prepared by dissolving sucrose (260 g) in Milli-Q water $(500 \mathrm{~mL})$. For the crystal growth, the sucrose solution $(10 \mathrm{~mL})$ was mixed with of CdTe QDs $(2.50 \mathrm{~mL})$ and the desired amount of Au NPs. In this study, samples including $0.125,0.50,0.75,1.00$, and $2.50 \mathrm{~mL}$ of $\mathrm{Au}$ NPs were employed. For the sake of simplicity in reading the article, we quote the volume of the added Au NP and CdTe QD into the crystallization mixture as the amounts used. In terms of particle numbers, the amount of QDs used in the study corresponds to $352.5 \mathrm{nmol}$ and the amounts of Au NPs used are 0.78, 1.56, 2.34, 3.13, and 15.6 pmol. The prepared mixture was dropped into a Petri dish (diameter $6 \mathrm{~cm}$ ) in an environment where the vibration is minimal. Vibration was further minimized using a 30 Duro Sorbothane vibration isolation pad. Within approximately 10 days, QD and Au NP embedded sucrose crystals were obtained. At the end of the 
crystallization, the whole surface of the Petri dish was covered with sucrose crystal pieces, laterally in centimeter scales. The thickness of the crystal formation was $\sim 2-3 \mathrm{~mm}$.

\subsection{Optical characterization}

The absorption measurements were carried out using a Cary 100 spectrophotometer. The quantum efficiency measurement of the crystals was carried out by placing the crystals on a blue LED (Avago ASMT) emitting at $460 \mathrm{~nm}$ and calculating the absorbed and emitted number of photons using an integrating sphere and a ZVision spectrophotometer. In solution quantum efficiency of the QDs were measured using Rhodamine 6G as the standard reference dye (whose quantum efficiency is $95 \%$ ). The absorbances of the $\mathrm{QD}$ and the reference dye were equated between 460 and $480 \mathrm{~nm}$ and below an absorbance of 0.1 to minimize the self-absorption effects. Subsequently, the emissions of QD and dye were collected using a Cary Eclipse fluorescence spectrophotometer by exciting at the wavelength where their absorbances were equated. The quantum efficiency was found by comparing the total number of photons emitted by the reference dye and the QDs. Time-resolved fluorescence spectra were obtained by employing a PicoHarp 200 time-resolved single photon counting system (PicoQuant) and the $1 / e$ value of the maximum photon count was reported as the lifetime.

\subsection{Structural characterization}

The crystal structure of the macrocrystals, which were finely powdered by using a mortar before the XRD measurement, was determined by a PANalytical X'Pert Pro Multi-purpose X-Ray Diffractometer operated at $45 \mathrm{kV}$ and $40 \mathrm{~mA}$ with $\mathrm{Cu} \mathrm{K} \alpha$ radiation and a diffracted beam monochromator. XRD spectra were taken between $10^{\circ}$ to $50^{\circ}$ of the $2 \theta$ angle with a step size of $0.01^{\circ}$.

The structural morphology of the as grown QD and $\mathrm{Au}$ NP embedded sucrose macrocrystals was further investigated using a FEI Quanta 200 FEG scanning electron microscope under high vacuum. The crystals were coated with $4 \mathrm{~nm}$ of $\mathrm{Au} / \mathrm{Pd}$ to minimize the charging of crystals before imaging.

QDs and Au NPs within the macrocrystals were imaged using a transmission electron microscope. In order to obtain thin sections, the macrocrystals were embedded into an Araldite 502 epoxy resin [20]. Following the curing of epoxy at $60{ }^{\circ} \mathrm{C}$, macrocrystal embedded epoxy was sectioned using a Leica ultramicrotome. Subsequently, sections in ethanol were transferred onto a lacey carbon coated 200 mesh copper grid and TEM images were recorded by a FEI Tecnai G2 F30 TEM.

\section{Acknowledgements}

We acknowledge ESF EURYI, EU-FP7 Nanophotonics4 Energy NoE, BMBF TUR 09/001, and TUBITAK EEEAG 109E002, 109E004, 110E010, 110E217, 112E183 and in part by NRF-CRP-6-2010-02 and NRF-RF2009-09. H. V. D. acknowledges additional support from TUBA-GEBIP and T. E. acknowledges support from TUBITAK BIDEB.

Electronic Supplementary Material: Supplementary material (comparison of Au NP dispersions in sucrose and in $\mathrm{NaCl}$ solution, details of Au NP and CdTe QD synthesis, absorption and photoluminescence spectra of as-synthesized CdTe QDs, absorption spectrum of $\mathrm{Au} \mathrm{NPs}$, and absorption spectrum of in water dissolved sucrose macrocrystal including $\mathrm{Au} \mathrm{NPs,}$ time-resolved fluorescence decays and lifetimes of CdTe QDs in the dispersions using which the crystallization is carried out, TEM image of the CdTe QDs and Au NPs in sucrose crystal without marking the nanoparticles, and photoluminescence lifetime of CdTe QD including macrocrystals) is available in the online version of this article at http://dx.doi.org/ 10.1007/s12274-014-0568-y.

\section{References}

[1] Nie, S. M.; Emory, S. R. Probing single molecules and single nanoparticles by surface-enhanced Raman scattering. Science 1997, 275, 1102-1106.

[2] Brolo, A. G. Plasmonics for future biosensors. Nat. Photon. 2012, 6, 709-713.

[3] Pelton, M.; Aizpurua, J.; Bryant, G. Metal-nanoparticle 
plasmonics. Laser Photon. Rev. 2008, 2, 136-159.

[4] Temnov, V. V. Ultrafast acousto-magneto-plasmonics. Nat. Photon. 2012, 6, 728-736.

[5] Ozel, T.; Hernandez Martinez, P. L.; Mutlugun, E.; Akin, O.; Nizamoglu, S.; Ozel, I. O.; Zhang, Q.; Xiong, Q. H.; Demir, $\mathrm{H}$. V. Observation of selective plasmon-exciton coupling in nonradiative energy transfer: Donor-selective versusacceptorselective plexcitons. Nano Lett. 2013, 13, 3065-3072.

[6] Xiao, M. D.; Jiang, R. B.; Wang, F.; Fang, C. H.; Wang, J. F.; Yu, J. C. Plasmon-enhanced chemical reactions. J. Mater. Chem. A 2013, 1, 5790-5805.

[7] Kauranen, M.; Zayats, A. V. Nonlinear plasmonics. Nat. Photon. 2012, 6, 737-748.

[8] Durach, M.; Rusina, A.; Stockman, M. I.; Nelson, K. Toward full spatiotemporal control on the nanoscale. Nano Lett. 2007, 7, 3145-3149.

[9] Israelowitz, M.; Amey, J.; Cong, T.; Sureshkumar, R. Spin coated plasmonic nanoparticle interfaces for photocurrent enhancement in thin film Si solar cells. J. Nanomater. 2014, $2014,639458$.

[10] Otto, T.; Müller, M.; Mundra, P.; Lesnyak, V.; Demir, H. V.; Gaponik, N.; Eychmuller, A. Colloidal nanocrystals embedded in macrocrystals: Robustness, photostability, and color purity. Nano Lett. 2012, 12, 5348-5354.

[11] Kalytchuk, S.; Zhovtiuk, O.; Rogach, A. L. Sodium chloride protected CdTe quantum dot based solid-state luminophores with high color quality and fluorescence efficiency. Appl. Phys. Lett. 2013, 103, 103105.
[12] Kim, Y.; Johnson, R. C.; Hupp, J. T. Gold nanoparticlebased sensing of "spectroscopically silent" heavy metal ions. Nano Lett. 2001, 1, 165-167.

[13] Teng, Y.; Ueno, K.; Shi, X.; Aoyo, D.; Qiu, J.; Misawa, H. Surface plasmon-enhanced molecular fluorescence induced by gold nanostructures. Ann. Phys. 2012, 524, 733-740.

[14] Albon, N.; Dunning, W. The observation of growth steps on sucrose crystals. ActaCryst. 1959, 12, 219-221.

[15] Govorov, A. O.; Bryant, G. W.; Zhang, W.; Skeini, T.; Lee, J.; Kotov, N. A.; Slocik, J. M.; Naik, R. R. Exciton-plasmon interaction and hybrid excitons in semiconductor-metal nanoparticle assemblies. Nano Lett. 2006, 6, 984-994.

[16] Rogach, A. L.; Franzl, T.; Klar, T. A.; Feldmann, J.; Gaponik, N.; Lesnyak, V.; Shavel, A.; Eychmüller, A.; Rakovich, Y. P.; Donegan, J. F. Aqueous synthesis of thiol-capped CdTenanocrystals: State-of-the-art. J. Phys. Chem. C 2007, 111, 14628-14637.

[17] Yu, W. W.; Qu, L. H.; Guo, W. Z.; Peng, X. G. Experimental determination of the extinction coefficient of CdTe, CdSe, and CdSnanocrystals. Chem. Mater. 2003, 15, 2854-2860.

[18] Frens, G. Controlled nucleation for the regulation of the particle size in monodisperse gold suspensions. Nature 1973, 241, 20-22.

[19] Haiss, W.; Thanh, N. T.; Aveyard, J.; Fernig, D. G. Determination of size and concentration of gold nanoparticles from UV-vis spectra. Anal. Chem. 2007, 79, 4215-4221.

[20] Glauert, A. Epoxy resins: An update on their selection and use. Microsc. Anal. 1991, 15-20. 